\title{
LAMC2 promotes the proliferation of cancer cells and induce infiltration of macrophages in non-small cell lung cancer
}

\author{
Meiyuan Liu ${ }^{1,2 \#} \wedge$, Rui Cai ${ }^{2 \#}$, Tian Wang ${ }^{1}$, Xia Yang $^{2}$, Meng Wang ${ }^{2}$, Zhongsheng Kuang ${ }^{3}$, Yuhui Xie ${ }^{3}$, \\ Jiren Zhang', Yanfang Zheng ${ }^{2}$ \\ ${ }^{1}$ Department of Oncology, Zhujiang Hospital of Southern Medical University, Guangzhou, China; ${ }^{2}$ Department of Medical Oncology, Affiliated \\ Cancer Hospital \& Institute of Guangzhou Medical University, Guangzhou, China; ${ }^{3}$ Department of Pathology, The First Affiliated Hospital of \\ Guangzhou Traditional Chinese Medicine University, Guangzhou, China \\ Contributions: (I) Conception and design: Y Zheng, J Zhang, M Liu, R Cai; (II) Administrative support: J Zhang, Y Zheng; (III) Provision of study \\ materials: Y Zheng, Z Kuang; (IV) Collection and assembly of data: T Wang, X Yang, M Wang; (V) Data analysis and interpretation: R Cai, M Liu, \\ Y Zheng, J Zhang, Y Xie, T Wang; (VI) Manuscript writing: All authors; (VII) Final approval of manuscript: All authors. \\ "These authors contributed equally to this work. \\ Correspondence to: Jiren Zhang. Department of Oncology, Zhujiang Hospital of Southern Medical University, Guangzhou, China. \\ Email: zhangiiren@126.com; Yanfang Zheng. Department of Medical Oncology, Affiliated Cancer Hospital \& Institute of Guangzhou Medical \\ University, Guangzhou, China. Email: 18665000236@163.com.
}

Background: Non-small-cell lung cancer (NSCLC) is the most prevalent cancer worldwide. Tumor microenvironment (TME) plays a very important role in the cancer development. Thus, it is urgent to find the change of TME that contributes to NSCLC carcinogenesis and progression.

Methods: The bioinformatics analysis approach was applied to evaluate the change of TME and screen the differentially immune cells in NSCLC tissue based on The Cancer Genome Atlas (TCGA) data. Meanwhile, the association of differentially immune cells with tumor stage and prognosis of NSCLC was evaluated. Then, we screen the different expression genes between macrophages infiltration high group and low group. After that, the expression of LAMC2 was detected in 48 cases of NSCLC tissues and paired normal tissues. The function of LAMC2 was detected through cell experiments in vitro. Immunohistochemistry assay was used to detect the correlation between LAMC2 expression and macrophages infiltration in NSCLC tissue. LAMC2-related pathways were identified by gene set enrichment analysis.

Results: Compared with early stage, middle-advanced stage of NSCLC exhibited lower immune score. Macrophages were the main component of different immune cells and correlated with poor outcome. The results of immunohistochemistry indicated that the expression of LAMC2 in NSCLC tissues was higher than paired normal tissues. Down-regulation of LAMC2 inhibited the proliferation, migration and invasion of NSCLC cells in vitro. Overexpression of LAMC2 was positively associated with macrophages infiltration in NSCLC tissues. Inhibition of LAMC2 expression in NSCLC cells could reduce THP-1 infiltration, and LAMC2 protein could promote the infiltration of THP-1. The Gene Set Enrichment Analysis results showed that high expression of $L A M C 2$ was correlated with focal adhesion and extracellular matrix receptor interaction.

Conclusions: Immune suppression and macrophages infiltration were correlated with poor outcomes in NSCLC. LAMC2 promoted macrophages infiltration and extracellular matrix remolding in NSCLC. Our studies suggested an oncogenic role of LAMC2 in NSCLC progression and it perhaps serve as a potential immune therapy target for NSCLC.

Keywords: LAMC2; macrophage; non-small-cell lung cancer (NSCLC); immune cell; tumor microenvironment (TME)

$\wedge$ ORCID: 0000-0002-9417-812X. 
Submitted Aug 17, 2021. Accepted for publication Sep 08, 2021.

doi: $10.21037 /$ atm-21-4507

View this article at: https://dx.doi.org/10.21037/atm-21-4507

\section{Introduction}

Worldwide, lung cancer is the most frequently occurring cancer in men and is the third most common cancer in women, and it is also the most common cause of cancerrelated death $(18.0 \%$ of the total cancer deaths) in both sexes (1). Non-small-cell lung cancer (NSCLC) accounts for $80-85 \%$ of lung cancers. In the past, the therapeutic efficacy of lung cancer was not satisfactory. Recently, the certain immune checkpoint blockers, such as antiprogrammed cell death protein 1 (PD-1) antibodies and cytotoxic T-lymphocyte-associated protein 4 (CTLA4) antibodies, had achieved promising results in NSCLC, but only a small part of the patients achieved good clinic outcome in these immunotherapies (2,3). This imply the presence of resistance, and the mechanisms need to be identified for more effective strategies can be proposed $(4,5)$.

Immune escape is one of the important reasons that affects the response rate of immunotherapy and is closely related to the change of tumor microenvironment (TME), which has been recognized as a key factor in several stages of disease progression, local resistance, metastasis and immune-escaping, thereby impact the treatment of oncology in the future (6). More and more studies on TME have been carried out, and it was found that TME contains a heterogeneous composition of immunosuppressive cells. Myeloid-derived suppressor cells (MDSCs) and tumorassociated macrophages (TAMs) alter the immune landscape of the TME and serve as facilitators of tumor proliferation, distant metastatic and immunotherapy resistance (7). TAMs, which can account for $50 \%$ of some solid tumors, constitute the heterogeneity and plasticity of tumor immune microenvironment (TIME) (8). Increasing evidence suggest that cytokines secreted by TAMs can induce anti-apoptotic, hyperproliferative and metastatic responses in lung cancer (9-11). TAMs can weaken the immunotherapy by impede $\mathrm{T}$ cells from reaching tumor cells (12). Targeting TAMs may treat as a possible therapeutic strategy to improve $\mathrm{T}$ cell interaction with cancer cells, thereby shifting "immuneexcluded" tumors, which are refractory to immune checkpoint inhibitors, into tumors that respond to antiPD-1 antibodies (12).
We used the bioinformatics analysis approach to screen the key genes accounting for TAMs infiltration. Laminin $\gamma 2$ (LAMC2) was one of the up-regulated genes that observed in TAMs rich NSCLC tissues. LAMC2 is the $\gamma 2$ subunit of the Heterotrimeric Glycoprotein Laminin-332(LAM-332, formerly known as Laminin-5, consisting of $\alpha 3, \beta 3$, and $\gamma 2$ chains). Although LAMC2 is recognized as an important structural component of the epithelial basement membrane in kinds of normal tissues, there is growing evidence that LAMC2 monomer plays a pathological role in cancers. High expression of LAMC2 can promotes invasion, migration and metastasis in human lung adenocarcinoma cells (13). And elevated serum level of LAMC2 is a poor prognostic factor of NSCLC (14).

However, the exact functions of LAMC2 in NSCLC TIME are still not fully understood. In the present study, we attempt to explore the significance of TAMs in NSCLC and further explore the underlying mechanisms of TAMS infiltration in NSCLC. We present the following article in accordance with the MDAR reporting checklist (available at https://dx.doi.org/10.21037/atm-21-4507).

\section{Methods}

\section{Imтипе cell infiltration analyses}

We acquired ImmuneScore, StromalScore of each sample in NSCLC through the ESTIMATE algorithm using the "estimate" package in $\mathrm{R}$ with default parameters. CIBERSORT algorithm could quantify the abundance of specific cell types (15). To compare the difference between early-stage NSCLC and middle-advanced NSCLC, the CIBERSORT analysis (https://cibersort.stanford.edu) was used to estimate the percentage of immune cells in each sample.

\section{Identification of macrophages infiltration-related DEGs}

The dataset between macrophages infiltration high groups and macrophages infiltration low groups was identified using Limma package in $\mathrm{R}$. The $\mathrm{P}<0.05$ and $\mid \log 2$ fold change I $\geq 1$ were set as the cut-off for the DEGs. The top 
50 DEGs were exhibited by the heatmap.

\section{Cell culture and conditioned media}

The human cell lines A549, H520, H1975, HCC827, PC9, H1299, BEAS-2B and THP-1 were authenticated by DNA short tandem repeat (STR) profiling. All cells were cultured in Dulbecco's Modified Eagle Medium (DMEM) supplemented with 10\% fetal bovine serum (FBS), $100 \mathrm{U} / \mathrm{mL}$ penicillin and $100 \mu \mathrm{g} / \mathrm{mL}$ streptomycin.

\section{$R N A$ interference}

Two small interfering RNAs (siRNAs) were designed to specifically target $L A M C 2$ : siRNA1: G T CAAA G C C T G T C C T T T GA; s i R A 2 : TCGGGAACTTCACAGACAA (RIBOBIO, Guangzhou, China). Non-targeting siNC as a negative control. The optimal conditions for transient transfection into NSCLC cell lines, determined in preliminary experiments, were $100 \mathrm{nmol} /$ well siRNA and $3 \mu \mathrm{L} /$ well Lipofectamine 3000 (ThermoFisher, USA) with $2 \mathrm{~mL}$ DMEM supplemented with $10 \%$ FBS in 6-well plate, and the cell culture medium was replaced after $24 \mathrm{~h}$. Cell samples were collected 30 or $48 \mathrm{~h}$ after transfection for qRT-PCR or Western blot. The experiments were biological replicated 4 times.

\section{Cell proliferation assay}

For CCK8 cell proliferation assay, H1975 and HCC827 cell proliferation was measured using a Cell Counting Kit (CCK8, BS350B, biosharp, China) according to the manufacturer's instruction. In brief, the cells $\left(1.5 \times 10^{3}\right.$ cells/ well with 4 wells in each group) were cultured in DMEM medium supplemented with $10 \%$ FBS for 1, 2, 3, 4, 5, 6 days and then $10 \mu \mathrm{L}$ of CCK8 reagent was added to each well. Cells were cultured in the dark for an additional $1 \mathrm{~h}$, and the absorbance at $450 \mathrm{~nm}$ was measured using a multifunctional enzyme labeling instrument (K3 Plus, BIODL). This experiment was biological replicated 3 times.

For EdU cell proliferation assay, we use BeyoClick ${ }^{\mathrm{TM}}$ EdU-555 cell proliferation Assay Kit (Cat\# C0075, Beyotime Biotechnology, China). According to the supplier's description, components are as follows: component 1, EdU; 2, Azide 555; 3, Click Reaction Buffer; 4, $\mathrm{CuSO}_{4}$; 5, Click Additive; 6, Hoechst 33342 (1,000×). 48 h after transfection with siRNA, tumor cells $\left(6 \times 10^{3}\right)$ were seeded in 96-well plates with 3 wells in each group and cultured overnight. Then the experimental procedure was carried according to the manufacturer's instruction. After the procedures completed, all nucleuses were stained blue, and the nucleuses of proliferating cells were stained red. The number of positive cells were obtained under fluorescence microscope and the proliferation rate was calculated. This experiment was biological replicated 3 times.

\section{$m R N A$ expression analysis}

RNA was isolated from cells using AG RNAex Pro Reagent AG21102 [Accurate Biotechnology (Human) Co., Ltd] following the manufacturer's instructions. And then reverse RNA into cDNA using Evo M-MLV RT Premix for qPCR AG11706 [Accurate Biotechnology (Human) Co., Ltd]. The cDNA was analyzed using real-time quantitative PCR (SYBR Green, Invitrogen) with an Applied SYBR Sequence Detection System (LightCycler 480 II, Roche). Each reaction was performed in triplicate and the experiment was biological replicated 3 times. The expression of each gene was normalized by human GAPDH.

Primers as follows:

GAPDH:

Forward primer: AATGGTGAAGGTCGGTGTGAACG; Reverse primer: TCGCTCCTGGAAGATGGTGATGG. $L A M C 2$ :

Forward primer: ACTGGAGCAGAAGCTTTCCC; Reverse primer: GTATTGTAGCAGCCTGGGGG.

\section{Immunobistochemistry analysis of NSCLC specimens}

The expression of LAMC2 and CD68 (the marker of macrophage) was determined by IHC from formalin-fixed, paraffin-embedded surgical specimens. All tumor specimens were obtained from which underwent complete surgical resection between 2019 and 2020 at Affiliated Cancer Hospital and Institute of Guangzhou Medical University with informed consent (Guangzhou, China). And this study was approved by the ethics committee of Affiliated Cancer Hospital and Institute of Guangzhou Medical University. Forty eight NSCLC specimens were chosen randomly from patients at $18-75$ years old. The study was conducted in accordance with the Declaration of Helsinki (as revised in 2013). Immunohistochemistry was performed using standard protocol. In brief, a pressure cooker $\left(95^{\circ} \mathrm{C}\right.$ for $30 \mathrm{~min}$ followed by $120^{\circ} \mathrm{C}$ for $10 \mathrm{~s}$ ) was used for antigen retrieval using antigen unmasking solution (Vector Laboratories). Antibodies specific to LAMC2 (BOSTER, \#BA3650), CD68 
(OriGene, \#ZM-0060) were used in this study.

\section{Western blotting}

Samples were subjected to SDS-PAGE on $10 \%$ polyacrylamide gels. After western transfer, the membranes were incubated with primary antibody LAMC2 $(1: 1,000)$ and internal reference antibody GAPDH (1:10,000. Then the blots were incubated with a secondary antibody (Invitrogen and Rockland). Signals were detected using the Tanon Infrared Imaging System (Guagnzhou EWELL BIO-TECHNOLOGY Co., LTD., China). This experiment was biological replicated 3 times.

\section{THP-1 migration assay}

For coculture: H1975 and HCC827 were planted into 24well plate $24 \mathrm{~h}$ after transfection, another $24 \mathrm{~h}$ later, the medium $(500 \mu \mathrm{L})$ was replaced and the 24-well Transwells ( $8 \mu \mathrm{m}$ pore size; Becton, Dickinson and Company, USA) were inserted to the plate. Then, THP-1 $\left(5 \times 10^{4}\right)$ were suspended in serum-free culture medium $(200 \mu \mathrm{L})$ with PMA $(100 \mathrm{ng} / \mathrm{mL})$ and seeded into inserts. For indicated factor: medium with LAMC2 protein (Cloud-Cline Corp.; Wuhan, China) was added to the 24-well plate, and THP-1 $\left(5 \times 10^{4}\right)$ were suspended in serum-free culture medium with PMA (100 ng/mL) and seeded into inserts. After $24 \mathrm{~h}$, the Transwells were taken out, and fixed with $4 \%$ paraformaldehyde for $15 \mathrm{~min}$, and then stained with crystal violet $(0.05 \%$, sigma) for $5 \mathrm{~min}$. The images of stained cells on the lower side were obtained under microscope. And this experiment was biological replicated 3 times.

\section{H1975 and HCC827 migration and invasion assay}

For migration assay: cell culture medium $(500 \mu \mathrm{L})$ with $10 \%$
FBS was added to the 24-well plate and the Transwells were inserted. H1975 $\left(3 \times 10^{4}\right)$ and HCC827 $\left(3 \times 10^{4}\right)$ cells $24 \mathrm{~h}$ after transfection of $L A M C 2$ siRNA were suspended in serumfree culture medium $(100 \mu \mathrm{L})$ and planted into the inserts. Another $24 \mathrm{~h}$ later, the inserts were taken out, and fixed with $4 \%$ paraformaldehyde for $15 \mathrm{~min}$, and then stained with crystal violet for $5 \mathrm{~min}$. The images of stained cells on the lower side were obtained under microscope. For invasion assay: the invasion assay followed a similar procedure to the migration assay, except that MATRIGEL MATRIX (BDBiocoat, America) was added to the inserts before the cells were seeded. The MATRIGEL MATRIX was diluted 20 times with cell culture medium on crushed ice according to instructions, and $100 \mu \mathrm{L}$ diluted MATRIGEL MATRIX was added in each insert. 2 h later, gelation was formed, and suspension of tumor cells was added. The following steps were same as in the migration experiment. And this experiment was biological replicated 3 times.

\section{Statistical analysis}

Student's $t$-test and Chi-square test were performed. Data are presented as mean \pm S.E.M. $\mathrm{P}<0.05$ was considered statistically significant: ${ }^{*} \mathrm{P}<0.05,{ }^{* *} \mathrm{P}<0.01$ and ${ }^{* * *} \mathrm{P}<0.001$.

\section{Results}

\section{Immune suppression and macrophage infiltration were related with poor prognosis of patients with NSCLC}

Using The Cancer Genome Atlas (TCGA) NSCLC datasets, we showed that the stage of NSCLC was correlated with poor outcomes (Figure 1A). Meanwhile, compared with early NSCLC, middle-advanced NSCLC exhibited lower immune signature but not stromal signature (Figure 1B). In order to further understand the change of TIME, we calculated the content of immune cells according to the
A

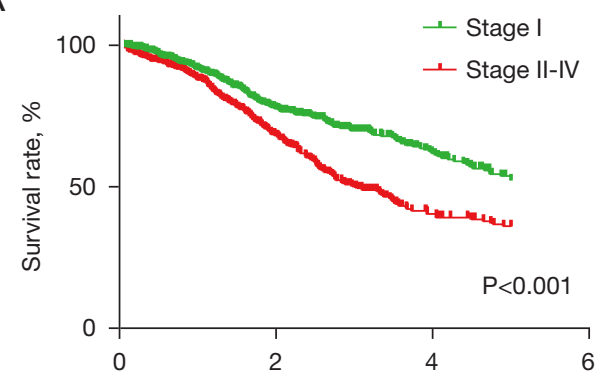

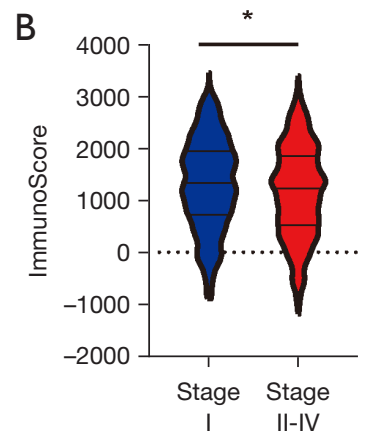

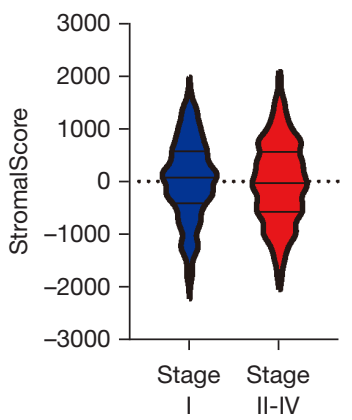




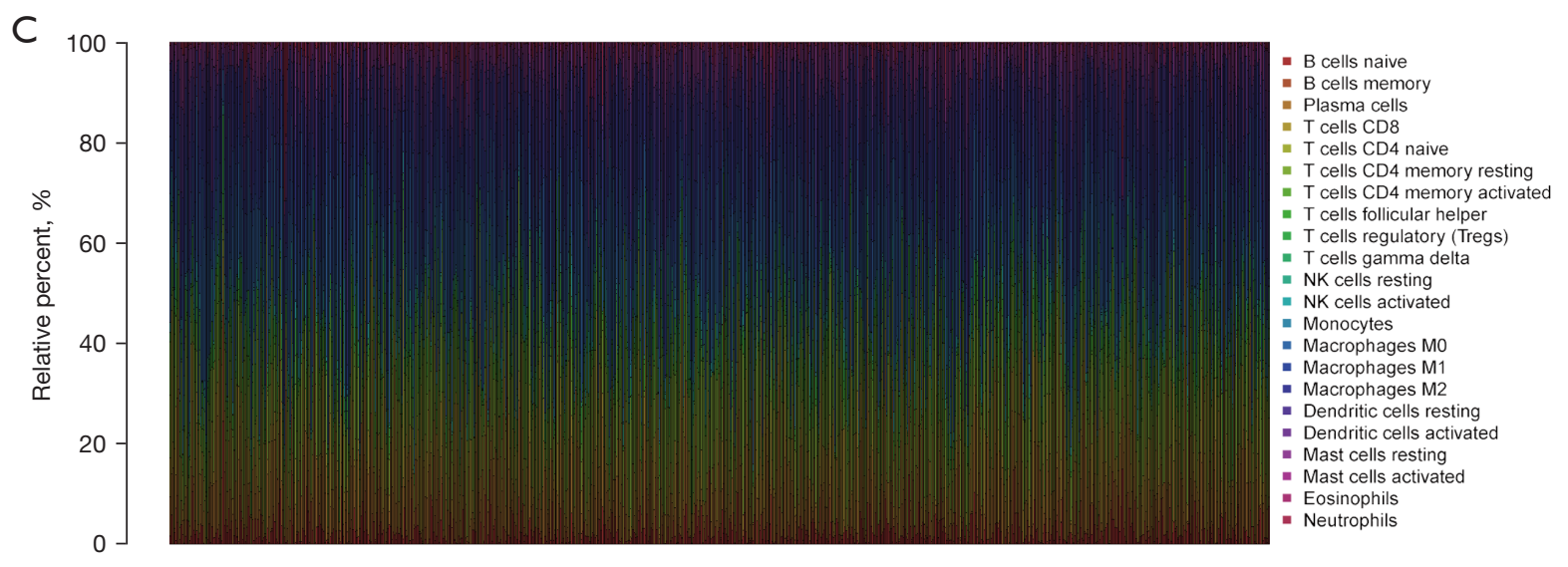

D
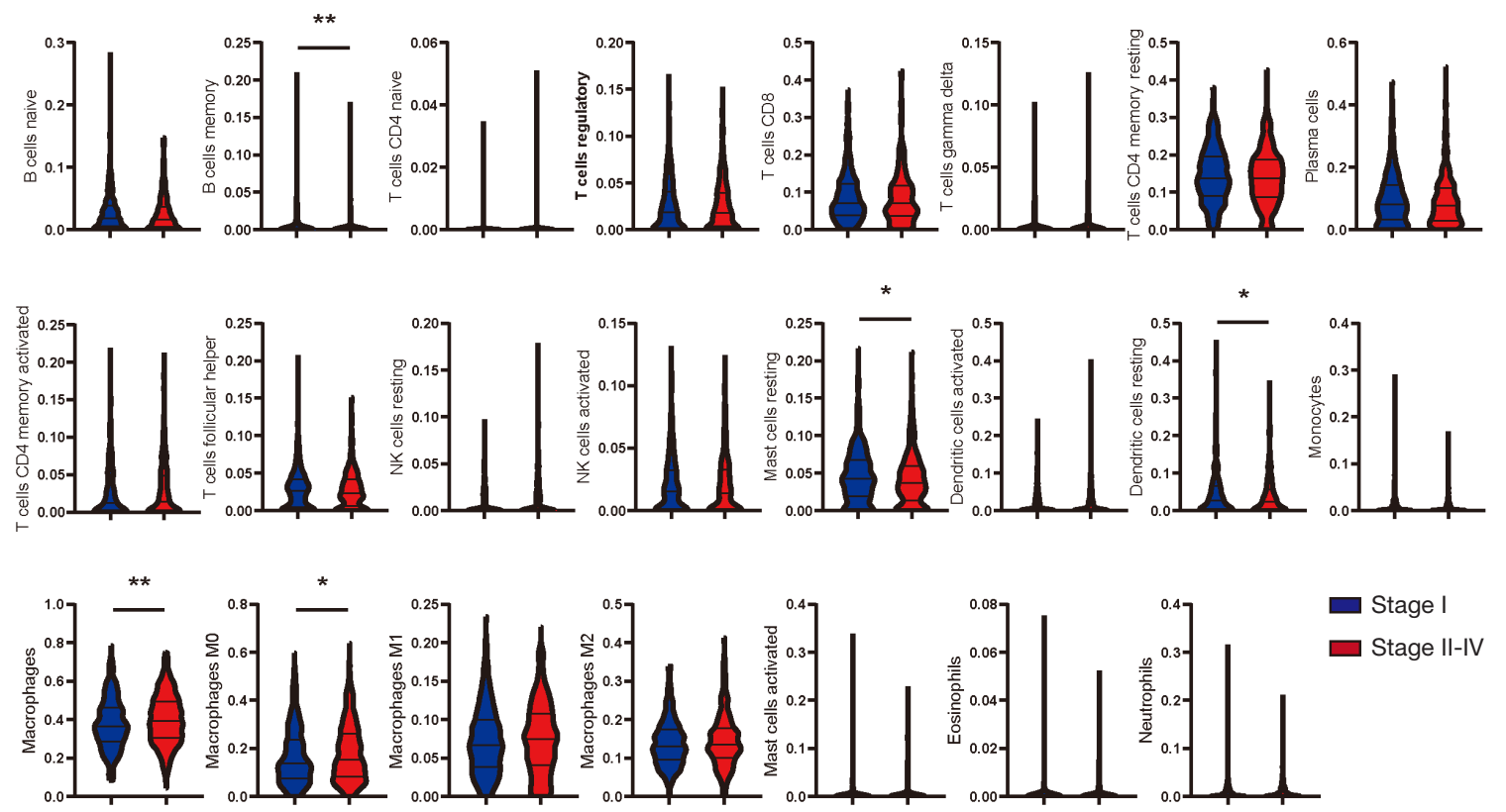

E
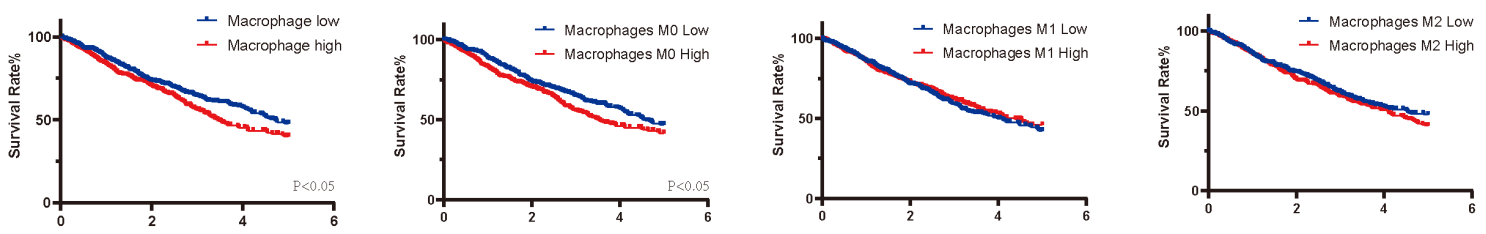

Figure 1 Immune analysis of NSCLC using TCGA NSCLC datasets. (A) Survival rate of stage I and stage II-IV of NSCLC. (B) Immune score and stromal score of stage I compare with stage II-IV. (C) Relative percent of different immune cells according to the CIBERSORT. (D) The percentage of various immune cells of stage I compared with stage II-IV in NSCLC. (E) The survival rate of different type of macrophages at different infiltration degree. * $\mathrm{P}<0.05 ;{ }^{* *}, \mathrm{P}<0.01$. NSCLC, non-small-cell lung cancer; TCGA, The Cancer Genome Atlas. 
CIBERSORT algorithm and displayed visually (Figure 1C). We further found that the content of B cells memory, mast cells resting and dendritic cells resting were decreased in advanced NSCLC compared with early NSCLC (Figure 1D). On the other hand, we found the content of macrophages and macrophages $\mathrm{M} 0$ were increased in advanced NSCLC compared with early NSCLC (Figure 1D). We detected that the content of macrophages was the most in differential immune cells and was correlated with poor outcomes (Figure 1D,1E). All the above results suggested that immune suppression and macrophage infiltration are related with poor prognosis of patients with NSCLC.

\section{LAMC2 was positively related to macrophages infiltration and poor prognosis of NSCLC}

TCGA datasets were used to explore the DEGs between macrophages low group and macrophages high group. A 2-fold change cutoff value was used for all ratios (ratio $>2.0$ ). The top 50 significant genes were displayed visually (Figure 2A). To characterize the functions of the different expression genes, we conducted GO enrichment analysis of molecular function (MF). The functions of DEGs were mainly involved in receptor ligand activity, glycosaminoglycan binding, hormone activity, extracellular matrix structural constituent and heparin binding (Figure 2B). To confirm the key genes, we analyze the relationship between top 50 significant genes and prognosis. The results suggesting that the expression of $L A M C 2$, APCDD1L and WNT7A were positively correlated with poor prognosis. $L A M C 2$ was the most significant $(\mathrm{P}<0.001)$ (Figure $2 C$ ).

We detected the expression of LAMC2 and the number of infiltrating macrophages in 48 cases of NSCLC tissues by IHC. CD68 was served as the marker of macrophages. We found that there was a positive correlation between LAMC2 expression and macrophages infiltration though they had different localization in NSCLC tissues. LAMC2 was mostly observed in cancer cells while macrophages were mainly located in the surrounding stroma (Figure 2D), and the expression of CD68 (the marker of macrophage) was positively correlated with LAMC2 by using Chi-square test (Table 1). Taken together, it was indicated that the overexpression of LAMC2 was positively associated with poor prognosis and macrophage infiltration in NSCLC.

\section{LAMC2 was overexpression in NSCLC}

The expression of LAMC2 protein was detected in 48 cases of NSCLC tissues and paired normal tissues by IHC (Figure $3 A$ ). The observations revealed that LAMC2 was mainly located in cancer cell cytoplasm, and the expression of LAMC2 was much higher than normal tissue in general. We further analyzed the expression of LAMC2 by GEPIA (Gene Expression Profiling Interactive Analysis) dataset (http://gepia.cancer-pku.cn/). The results showed that the expression of LAMC2 was upregulated in lung cancer tissues (Figure 3B). Using the methods of $\mathrm{qRT}$-PCR and $\mathrm{WB}, \mathrm{LAMC} 2$ was found significantly overexpressed in several NSCLC cell lines compared with normal bronchoalveolar cell line (BEAS-2B) (Figure 3C,3D). All the above results suggested that the expression of LAMC2 was upregulated in NSCLC cells.

\section{Knockdown of LAMC2 inbibited proliferation of NSCLC cells}

The expression of $L A M C 2$ was knocked-down by siRNA in H1975 and HCC827 cells and the silencing efficiency was evaluated using qRT-PCR and Western blot (Figure 4A,4B). The result showed that LAMC2-inhibited cells had reduced proliferation abilities when compared to the control cells as detected by CCK-8 (Figure 4C), EdU assay demonstrated that less EdU positive cells were observed in LAMC2inhibited cells than control group (Figure 4D). All the above results suggested that LAMC2 may promote NSCLC cells proliferation in vitro.

\section{Knockdown of LAMC2 inbibited migration and invasion of NSCLC cells}

In order to validate if LAMC2 has effect on the migration and invasion of NSCLC cells, we conducted Transwell assay. The result showed that LAMC2-inhibited cells had reduced migration (Figure $5 A$ ) and invasion (Figure $5 B$ ) abilities when compared to the control cells. The results suggested that LAMC2 may promote the migration and invasion of NSCLC cells in vitro.

\section{LAMC2 promoted macrophages infiltration by ECM remodeled}

To validate LAMC2 as a macrophage chemoattractant in vitro, we conducted the transwell assay. The results showed that condition medium (CM) from LAMC2-inhibited NSCLC cells induced significantly less macrophage migration than CM from negative control cells (Figures 6A). Meanwhile 

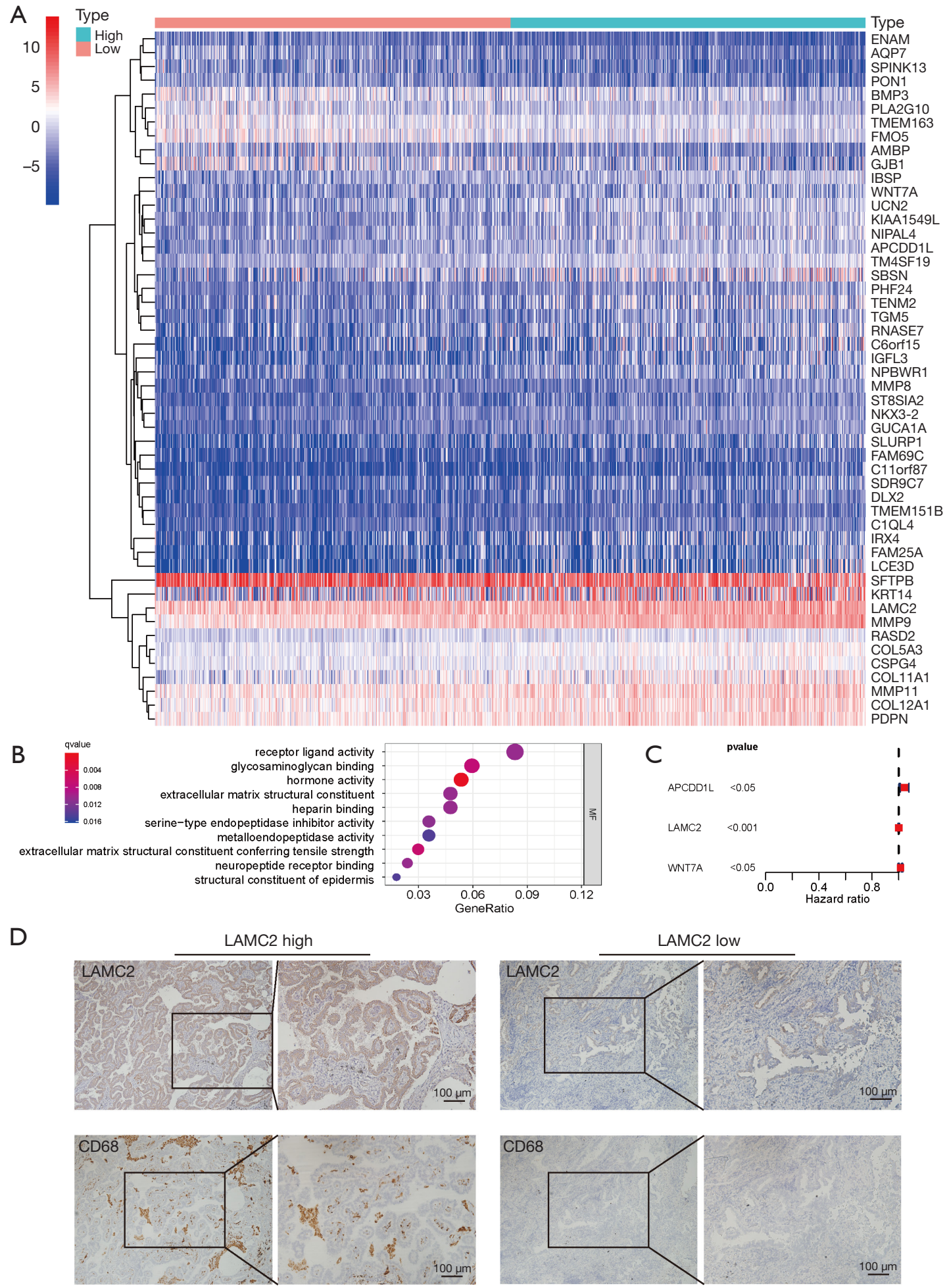

Figure 2 The relationship of LAMC2 with different degree of macrophage. (A) The analysis of DEGs between macrophages low group and macrophages high group using TCGA datasets. (B) GO enrichment analysis of MF. (C) The analysis of the genes that were correlated with poor prognosis in top 50 significant genes. (D) The expression of LAMC2 and CD68 in 48 cases of NSCLC tissues detected by IHC. DEGs, Differentially Expressed Genes; TCGA, The Cancer Genome Atlas; GO, Gene Ontology; MF, molecular function; NSCLC, nonsmall-cell lung cancer; IHC, immunohistochemistry. 
Table 1 Correlation between the expression of LAMC2 and CD68 by Chi-square test

\begin{tabular}{lcccc}
\hline & CD68-high & CD68-low & $\chi^{2}$ & $\mathrm{P}$ \\
\hline LAMC2-high & 18 & 3 & 8.584 & 0.003 \\
LAMC2-low & 12 & 15 & & \\
\hline
\end{tabular}

recombinant LAMC2-supplemented media increased macrophage migration (Figures 6B). The Gene Set Enrichment Analysis (GSEA) in TCGA NSCLC patients showed that LAMC2 promotes focal adhesion and ECM receptor interaction, which indicated extracellular matrix is remodeled (Figure 6C). Thus, LAMC2 functions as a potent
A

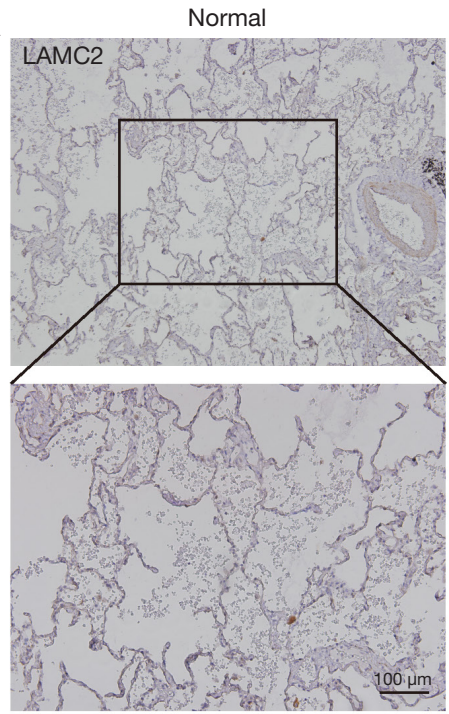

B

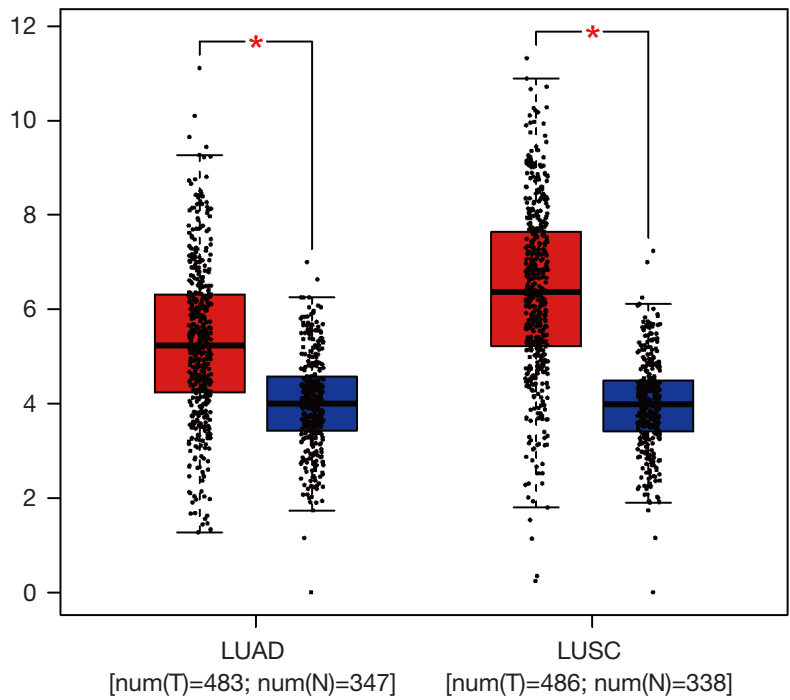

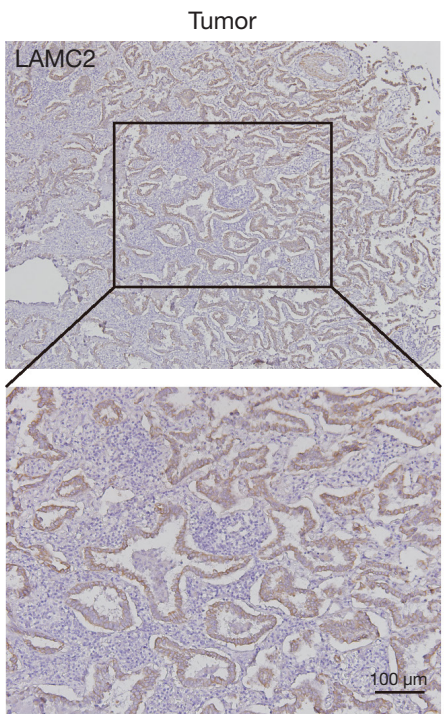

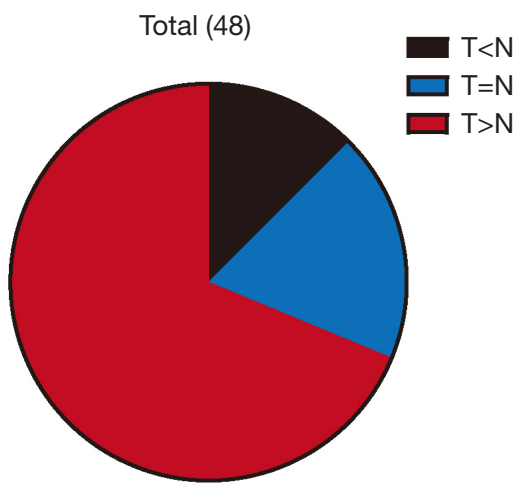

C
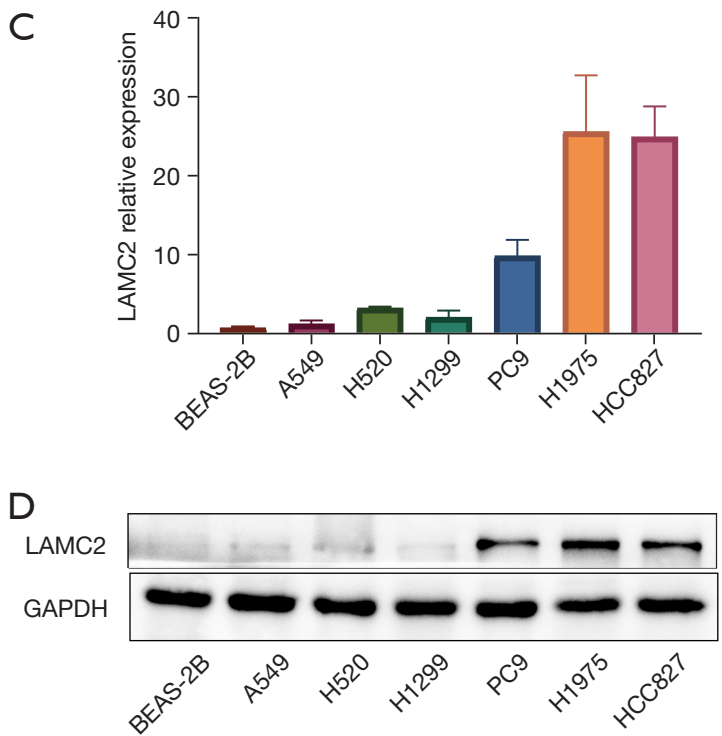

Figure 3 The expression of LAMC2. (A) The expression of LAMC2 protein and paired normal tissues detected by IHC. The right pie chart showed the percentage of different level of LAMC2 (Red part: tumor's LAMC2 expression was higher than normal tissue, $68.75 \%$; Blue part: tumor's LAMC2 expression is approximate to normal tissue, $18.75 \%$; Black part: tumor's LAMC2 expression is lower than normal tissue, 12.5\%). (B) The expression of $L A M C 2$ analyzed by GEPIA dataset. (C,D) The expression of LAMC2 in several NSCLC cell lines compared with normal bronchoalveolar cell line (BEAS-2B) detected by qRT-PCR and Western blot. *, P<0.05. IHC, immunohistochemistry; GEPIA, Gene Expression Profiling Interactive Analysis; NSCLC, non-small-cell lung cancer; qRT-PCR, quantitative real time polymerase chain reaction. 
A
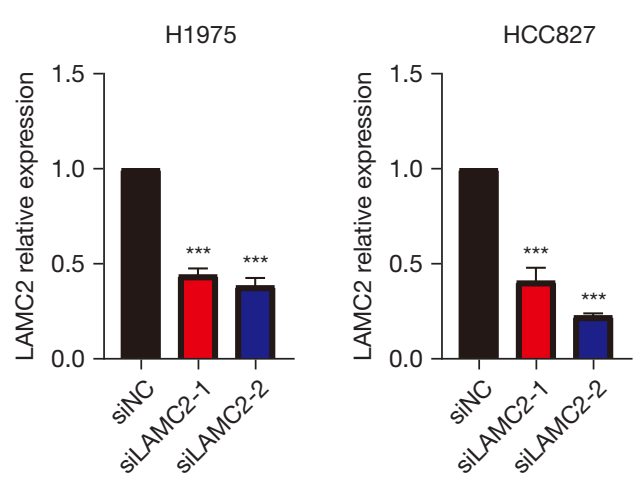

B

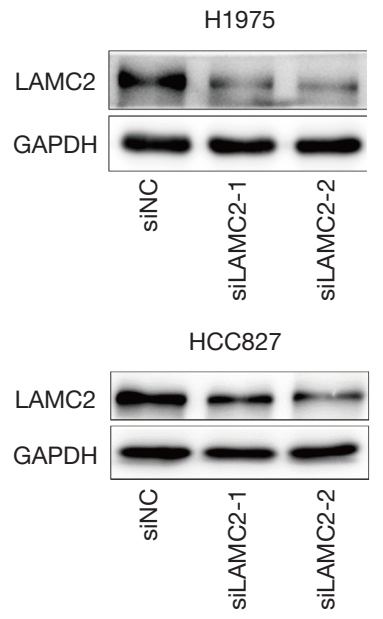

C

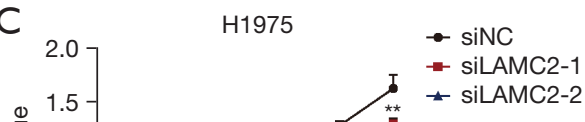

D
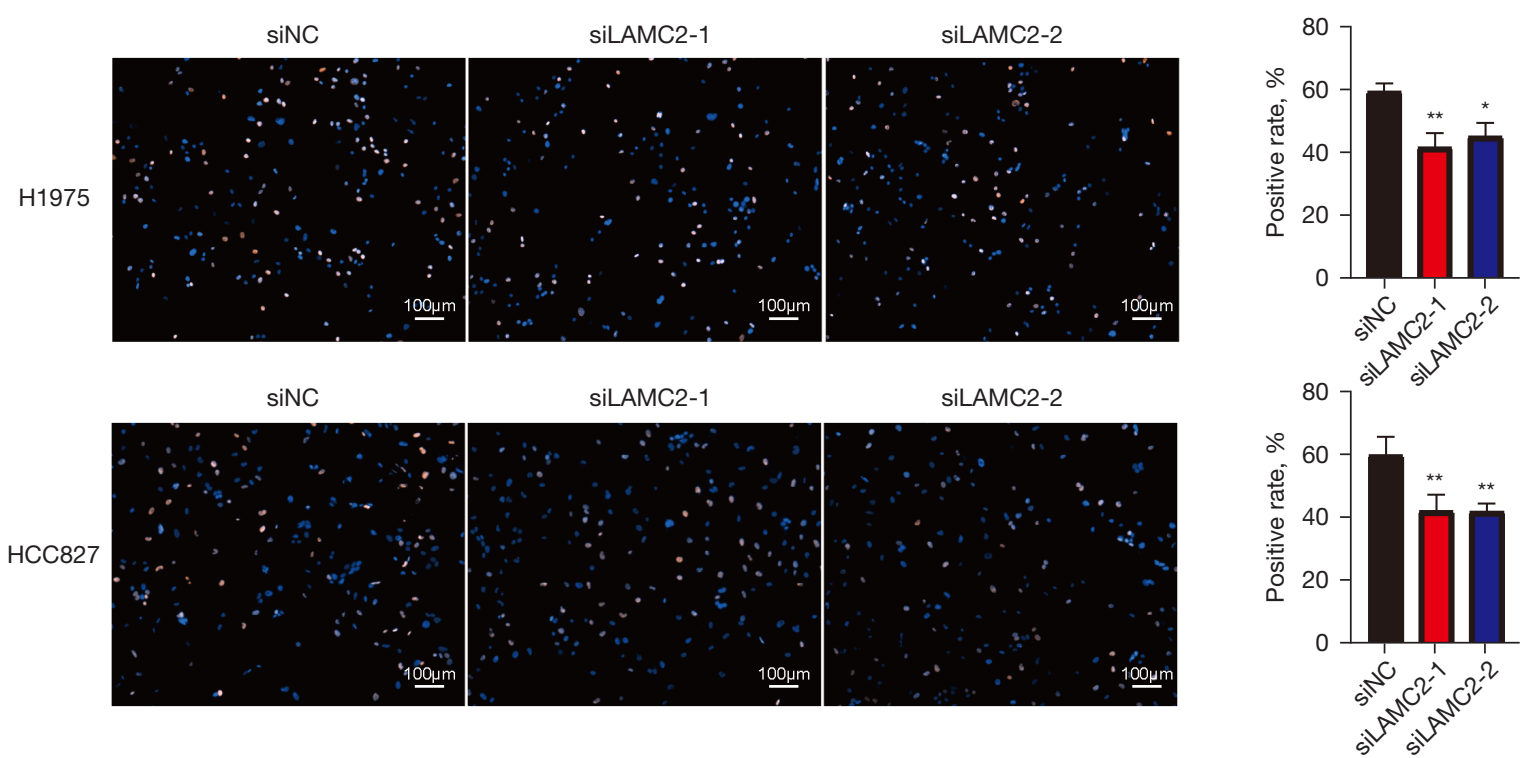

Figure 4 LAMC2 promoted NSCLC cells proliferation. (A,B) The expression of LAMC2 in H1975 and HCC827 cell lines after LAMC2 siRNA transfection detected by qRT-PCR and Western blot. (C) The proliferation of H1975 and HCC827 after knockdown of LAMC2 detected by CCK-8. (D) EdU-labeled proliferating cells of H1975 and HCC827 after transfection of LAMC2 siRNA by using EdU assay. *, $\mathrm{P}<0.05 ;{ }^{* *}, \mathrm{P}<0.01 ;{ }^{* * *}, \mathrm{P}<0.001$. NSCLC, non-small-cell lung cancer; siRNA, small interfering Ribonucleic Acid; qRT-PCR, quantitative real time polymerase chain reaction.

chemokine for the recruitment of macrophages to the NSCLC TME.

\section{Discussion}

Lung cancer is the first leading cause of cancer-related deaths worldwide. About $85 \%$ lung cancer is classified as NSCLC (16). Middle-advanced NSCLC patients always exhibited poor prognosis $(16,17)$. Accumulating evidence forecasts that the tumor-stroma crosstalk promotes cancer progression by modulating tumor malignancy, TME remodeling and inducing immune evasion $(8,18)$. Although immune checkpoint inhibitors (ICIs) have achieved promising results in several malignancies, the resistance remains an issue. The imbalance of immuno-metabolism plays a dominating role in the TME. An increased concentration of extracellular adenosine, the depletion of tryptophan and uncontrolled activation of the PI3K/AKT 
A
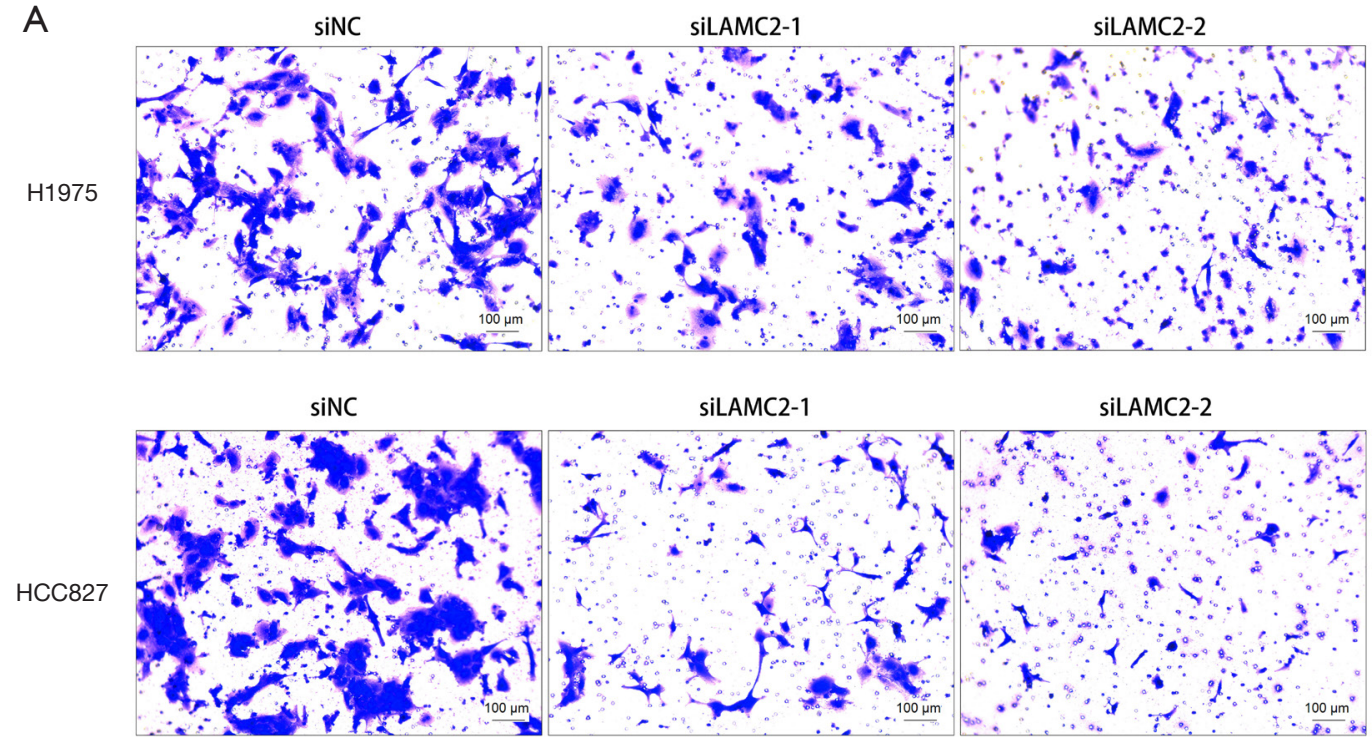

B

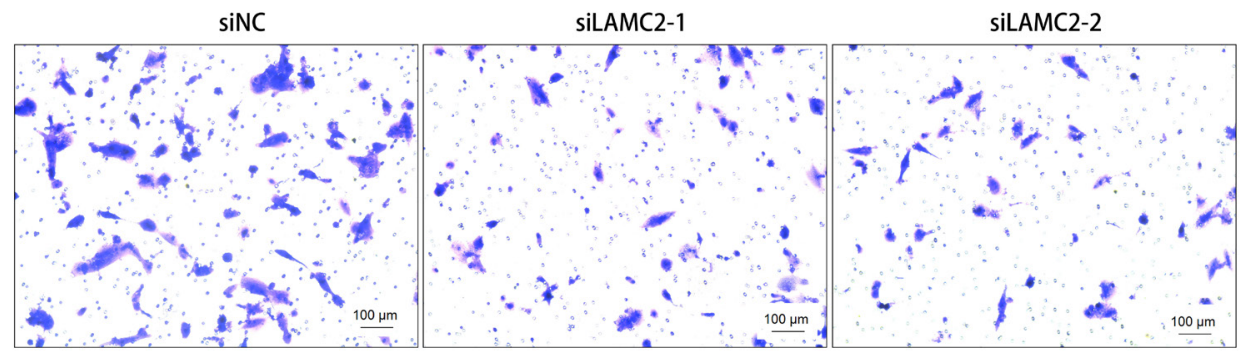

$\mathrm{H} 1975$
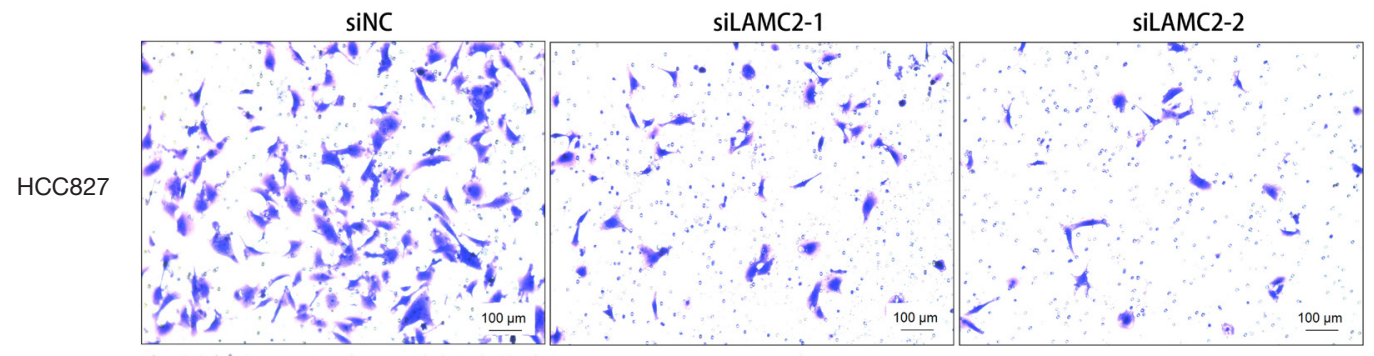

SiLAMC2-1

SiLAMC2-2
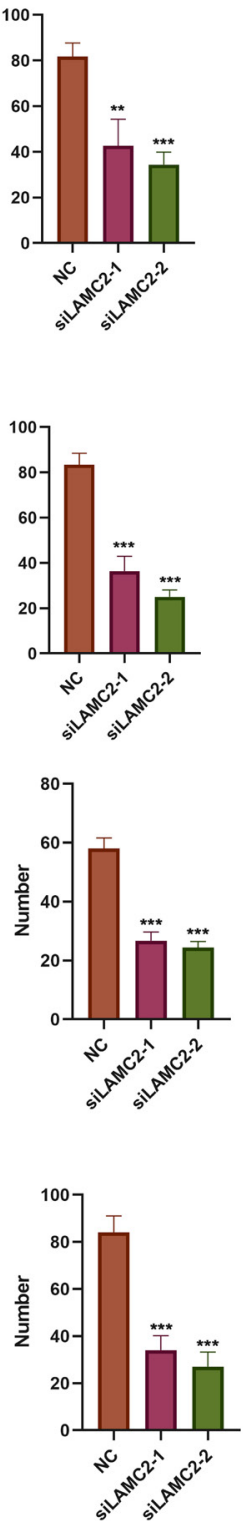

Figure 5 LAMC2 promoted NSCLC cells migration and invasion. (A) The migration of H1975 and HCC827 after knockdown of LAMC2 detected by Transwell migration assay. (B) The invasion of H1975 and HCC827 after knockdown of LAMC2 detected by Transwell invasion assay. NSCLC, non-small-cell lung cancer. ${ }^{* *}, \mathrm{P}<0.01 ;{ }^{* * *}, \mathrm{P}<0.001$. NSCLC, non-small-cell lung cancer.

pathway induce an immune-tolerant TME. Additionally, abnormal angiogenesis induces a hypoxic environment by recruiting VEGF, Treg cells and TAMs to reduce the response to ICIs (19). So, new strategies may include targeting the adenosine pathway and immune-suppressive cells. To overcome the resistance of ICIs in NSCLC, it is important to identify the change of TME at first.

In this study, we explored the change of TME in NSCLC progression. With tumor progression we found the immune score was decreased, but not stroma score. It indicated that immunosuppressive microenvironment plays a central role in tumor progression. In order to find how the immunosuppressive microenvironment was formed. We used the bioinformatics analysis approach to screen different immune cells between early stage of NSCLC and middle-advanced stage of NSCLC. Moreover, we found that macrophages which account for up to $50 \%$ of immune cells were the most components in TME and were related 
A
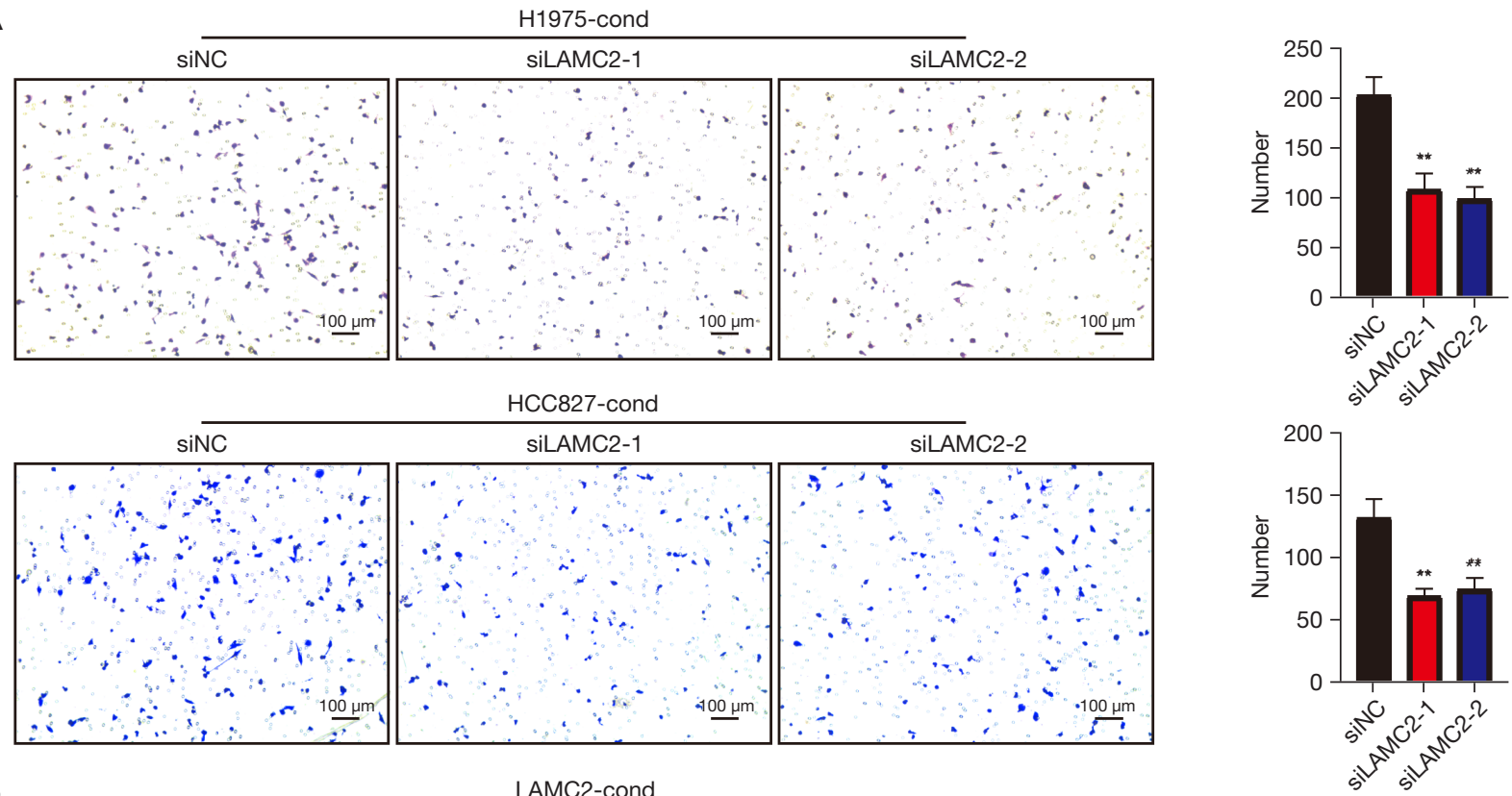

B
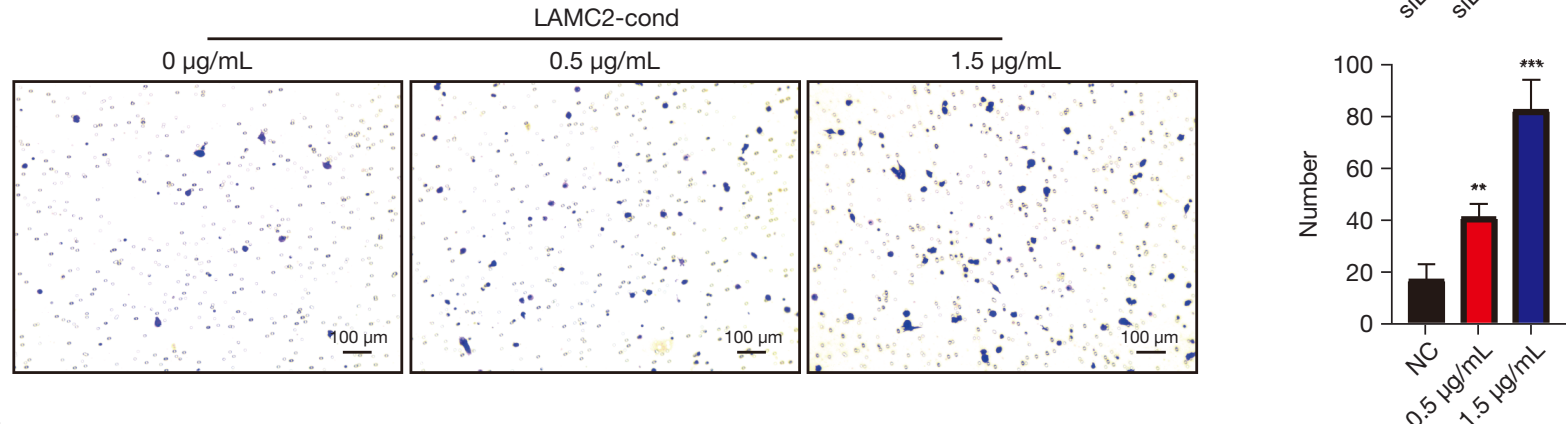

C Enrichment plot: KEGG ECM RECEPTOR INTERACTION
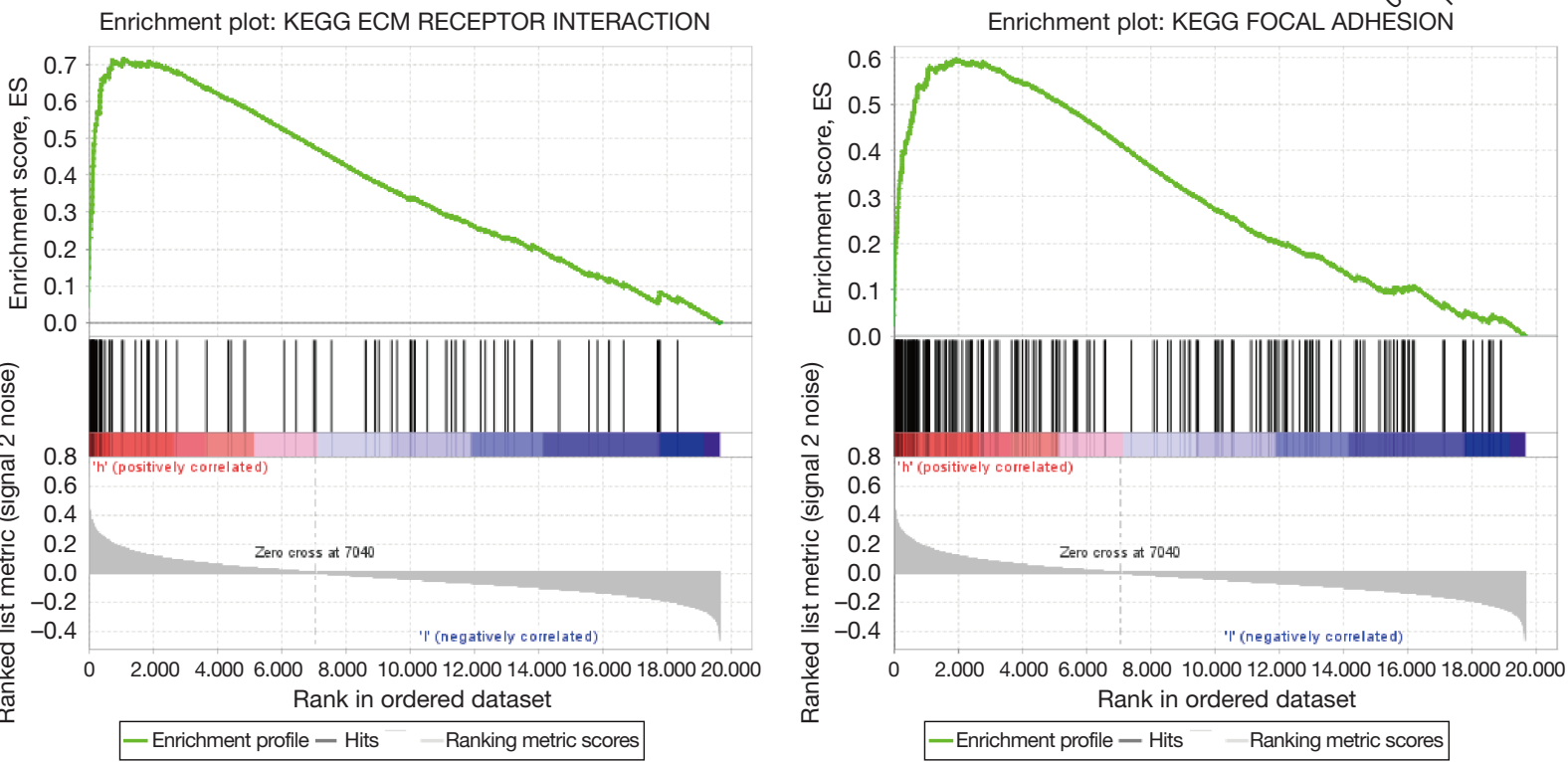

Figure 6 The influence of LAMC2 on the migration of THP-1. (A) Migration assay of THP-1 when coculture with LAMC2-knockdown H1975 and HCC827. (B) Migration assay of THP-1 upon addition of LAMC2-supplemented media. (C) The function of LAMC2 analyzed by the GSEA in TCGA NSCLC patients. ${ }^{* *}, \mathrm{P}<0.01$; ${ }^{* * *}, \mathrm{P}<0.001$. GSEA, Gene Set Enrichment Analysis; TCGA, The Cancer Genome Atlas; NSCLC, non-small-cell lung cancer. 
with poor prognosis of NSCLC patients, that is consistent with previous findings (20). Notably, M1 and M2 type macrophages are not exhibited any difference. Importantly, the interaction between TAMs and cancer cells are essential for the maintenance of immunosuppression in the TME. TAMs released some cytokine to inhibit anticancer immunity cells, for example helper CD4+ T cells, cytotoxic CD8+ T cells, and natural killer (NK) cells (8). Even though the original hypotheses proposed that macrophages are involved in antitumor immunity, there is abundant of clinical and experimental evidence that TAMs may promote tumor progression by promoting tumor proliferation, invasion and metastasis $(21,22)$. The number of TAMs is significantly associated with angiogenesis and lymphangiogenesis in NSCLC TME $(23,24)$. Our above studies implied that TAMs involved in NSCLC progression by inhibiting immune response.

Next, we explored the mechanisms underlying the recruitment of TAMs. Our bioinformatics analysis found that the top 50 different genes in macrophage high NSCLC tissues were mainly involved in receptor ligand activity and extracellular matrix structural constituent. Three of the differential genes (APCDD1, LAMC2, WNT7A) were closely related to the poor prognosis of NSCLC, and $L A M C 2$ was the most significant one.

LAMC2 is an important structural component of ECM (25). Our study revealed that the expression of LAMC2 was higher in epithelial-stromal interface of tumor clusters than that in paired normal tissues, which is consistent with previous outcomes (26,27). Meanwhile, we also found that LAMC2 was overexpressed in NSCLC cells compared with alveolar epithelial cell. Our research also showed that down-regulation of LAMC2 inhibited proliferative, migration and invasion properties of NSCLC cells. Thus, we suggested that LAMC2 may act as an oncogene during NSCLC progression. Combine the result of LAMC2 GSEA in our study with previous studies, it may be concluded that the mechanism of LAMC2 on NSCLC is that the elevated LAMC2 in cancer cells may, through interaction with integrin $\beta 1$, hijack part of the focal adhesion complex including FAK/SRC kinase to induce EMT via ZEB1 and/or Snail $(13,28)$.

In further research, we first investigated whether the expression of LAMC2 in tumor cells could affect infiltration of macrophages. It has been reported that secreted LAMC2 in the conditioned medium promotes lung cancer cell migration and invasion (13), but the effect of LAMC2 on macrophages has not been mentioned. In our study, we found that high infiltration of macrophages was observed in corresponding areas of high LAMC2 expression in NSCLC tissues. Meanwhile, silencing LAMC2 in NSCLC cells could inhibit macrophages migration and recombination LAMC2 could promoted macrophages migration. It exhibited that LAMC2 can act as a chemoattractant to promote macrophages infiltration. Our results also show that high expression of LAMC2 activated ECM receptor interaction and focal adhesion signaling pathways. The activation of two signaling pathways activated is an essential event in tumor development and is associated with increased extracellular matrix adhesion and cell motility $(29,30)$. Together, our work certifies the view that LAMC2 can act as a potent chemokine produced by NSCLC cells to attract macrophages into NSCLC TME and promote TME ECM remolding.

In conclusion, our findings showed that middle-advanced NSCLC exhibited immune suppression and macrophages infiltration involved in formation of immunosuppressive TME. Furthermore, LAMC2 promoted macrophages infiltration and ECM remolding in NSCLC tissues. However, there is a limitation that the involved signaling pathway have not yet been fully elucidated in this study and further study is required.

In general, our study suggested that LAMC2 may supply a potential immune therapy target for NSCLC.

\section{Acknowledgments}

Funding: This work was supported by grant from Clinical Key Specialty Construction Project of Guangzhou Medical University (No. YYPT202017), grant from Guangzhou High-Level Clinical Key Specialty Construction Project (No. 2019-2021), and grant from Scientific Project Foundation of Guangzhou City (No. 201907010037).

\section{Footnote}

Reporting Checklist: The authors have completed the MDAR reporting checklist. Available at https://dx.doi. org/10.21037/atm-21-4507

Data Sharing Statement: Available at https://dx.doi. org/10.21037/atm-21-4507

Conflicts of Interest: All authors have completed the ICMJE uniform disclosure form (available at https://dx.doi. org/10.21037/atm-21-4507). The authors have no conflicts 
of interest to declare.

Ethical Statement: The authors are accountable for all aspects of the work in ensuring that questions related to the accuracy or integrity of any part of the work are appropriately investigated and resolved. All tumor specimens were obtained from which underwent complete surgical resection between 2019 and 2020 at Affiliated Cancer Hospital and Institute of Guangzhou Medical University with informed consent (Guangzhou, China). And this study was approved by the ethics committee of Affiliated Cancer Hospital and Institute of Guangzhou Medical University. The study was conducted in accordance with the Declaration of Helsinki (as revised in 2013).

Open Access Statement: This is an Open Access article distributed in accordance with the Creative Commons Attribution-NonCommercial-NoDerivs 4.0 International License (CC BY-NC-ND 4.0), which permits the noncommercial replication and distribution of the article with the strict proviso that no changes or edits are made and the original work is properly cited (including links to both the formal publication through the relevant DOI and the license). See: https://creativecommons.org/licenses/by-nc-nd/4.0/.

\section{References}

1. Sung H, Ferlay J, Siegel RL, et al. Global Cancer Statistics 2020: GLOBOCAN Estimates of Incidence and Mortality Worldwide for 36 Cancers in 185 Countries. CA Cancer J Clin 2021;71:209-49.

2. Chae YK, Arya A, Iams W, et al. Current landscape and future of dual anti-CTLA4 and PD-1/PD-L1 blockade immunotherapy in cancer; lessons learned from clinical trials with melanoma and non-small cell lung cancer (NSCLC). J Immunother Cancer 2018;6:39.

3. Seidel JA, Otsuka A, Kabashima K. Anti-PD-1 and AntiCTLA-4 Therapies in Cancer: Mechanisms of Action, Efficacy, and Limitations. Front Oncol 2018;8:86.

4. Anderson KG, Stromnes IM, Greenberg PD. Obstacles Posed by the Tumor Microenvironment to T cell Activity: A Case for Synergistic Therapies. Cancer Cell 2017;31:311-25.

5. Chen DS, Mellman I. Elements of cancer immunity and the cancer-immune set point. Nature 2017;541:321-30.

6. Chen F, Zhuang X, Lin L, et al. New horizons in tumor microenvironment biology: challenges and opportunities. BMC Med 2015;13:45.
7. Osipov A, Saung MT, Zheng L, et al. Small molecule immunomodulation: the tumor microenvironment and overcoming immune escape. J Immunother Cancer 2019;7:224.

8. Vitale I, Manic G, Coussens LM, et al. Macrophages and Metabolism in the Tumor Microenvironment. Cell Metab 2019;30:36-50.

9. Schmall A, Al-Tamari HM, Herold S, et al. Macrophage and cancer cell cross-talk via CCR2 and CX3CR1 is a fundamental mechanism driving lung cancer. Am J Respir Crit Care Med 2015;191:437-47.

10. Pullamsetti SS, Savai R, Seeger W, et al. Translational Advances in the Field of Pulmonary Hypertension. From Cancer Biology to New Pulmonary Arterial Hypertension Therapeutics. Targeting Cell Growth and Proliferation Signaling Hubs. Am J Respir Crit Care Med 2017;195:425-37.

11. Weichand B, Popp R, Dziumbla S, et al. S1PR1 on tumor-associated macrophages promotes lymphangiogenesis and metastasis via NLRP3/IL-1 . J Exp Med 2017;214:2695-713.

12. Peranzoni E, Donnadieu E. Improving efficacy of cancer immunotherapy through targeting of macrophages. Hum Vaccin Immunother 2019;15:189-92.

13. Moon YW, Rao G, Kim JJ, et al. LAMC2 enhances the metastatic potential of lung adenocarcinoma. Cell Death Differ 2015;22:1341-52.

14. Korbakis D, Dimitromanolakis A, Prassas I, et al. Serum LAMC2 enhances the prognostic value of a multiparametric panel in non-small cell lung cancer. $\mathrm{Br} \mathrm{J}$ Cancer 2015;113:484-91.

15. Newman AM, Liu CL, Green MR, et al. Robust enumeration of cell subsets from tissue expression profiles. Nat Methods 2015;12:453-7.

16. Thai AA, Solomon BJ, Sequist LV, et al. Lung cancer. Lancet 2021;398:535-54.

17. Postmus PE, Kerr KM, Oudkerk M, et al. Early and locally advanced non-small-cell lung cancer (NSCLC): ESMO Clinical Practice Guidelines for diagnosis, treatment and follow-up. Ann Oncol 2017;28:iv1-iv21.

18. Wu Z, Li S, Zhu X. The Mechanism of Stimulating and Mobilizing the Immune System Enhancing the AntiTumor Immunity. Front Immunol 2021;12:682435.

19. Giannone G, Ghisoni E, Genta S, et al. ImmunoMetabolism and Microenvironment in Cancer: Key Players for Immunotherapy. Int J Mol Sci 2020;21:4414.

20. Larionova I, Tuguzbaeva G, Ponomaryova A, et al. TumorAssociated Macrophages in Human Breast, Colorectal, 


\section{Page 14 of 14}

Lung, Ovarian and Prostate Cancers. Front Oncol 2020;10:566511.

21. Chen Y, Song Y, Du W, et al. Tumor-associated macrophages: an accomplice in solid tumor progression. J Biomed Sci 2019;26:78.

22. Liang ZW, Ge XX, Xu MD, et al. Tumor-associated macrophages promote the metastasis and growth of nonsmall-cell lung cancer cells through NF- $\mathrm{B} / \mathrm{PP} 2 \mathrm{Ac}-$ positive feedback loop. Cancer Sci 2021;112:2140-57.

23. Arenberg DA, Keane MP, DiGiovine B, et al. Macrophage infiltration in human non-small-cell lung cancer: the role of CC chemokines. Cancer Immunol Immunother 2000;49:63-70.

24. Hwang I, Kim JW, Ylaya K, et al. Tumor-associated macrophage, angiogenesis and lymphangiogenesis markers predict prognosis of non-small cell lung cancer patients. J Transl Med 2020;18:443.

25. Colognato H, Yurchenco PD. Form and function: the laminin family of heterotrimers. Dev Dyn 2000;218:213-34.

Cite this article as: Liu M, Cai R, Wang T, Yang X, Wang M, Kuang Z, Xie Y, Zhang J, Zheng Y. LAMC2 promotes the proliferation of cancer cells and induce infiltration of macrophages in non-small cell lung cancer. Ann Transl Med 2021;9(17):1392. doi: 10.21037/atm-21-4507

\section{Liu et al. LAMC2 induces infiltration of macrophages in NSCLC}

26. Määttä M, Soini Y, Pääkkö P, et al. Expression of the laminin gamma2 chain in different histological types of lung carcinoma. A study by immunohistochemistry and in situ hybridization. J Pathol 1999;188:361-8.

27. Hamasaki H, Koga K, Aoki M, et al. Expression of laminin $5-\gamma 2$ chain in cutaneous squamous cell carcinoma and its role in tumour invasion. Br J Cancer 2011;105:824-32.

28. Marinkovich MP. Tumour microenvironment: laminin 332 in squamous-cell carcinoma. Nat Rev Cancer 2007;7:37080.

29. Masi I, Caprara V, Bagnato A, et al. Tumor Cellular and Microenvironmental Cues Controlling Invadopodia Formation. Front Cell Dev Biol 2020;8:584181.

30. Seetharaman S, Etienne-Manneville S. Cytoskeletal Crosstalk in Cell Migration. Trends Cell Biol 2020;30:720-35.

(English Language Editor: J. Chapnick) 